\title{
Calendar
}

\section{National Meetings}

\section{APA}

American Psychological Association

- August 7-10, 2003, Toronto, Ontario, Canada

- July 28-August 1, 2004, Honolulu, HI

- August 18-21, 2005, Washington, DC

- August 10-13, 2006, New Orleans, LA

- August 16-19, 2007, San Francisco, CA

For information

APA Convention Office

750 First Street, NE

Washington, DC 20002-4242

Telephone: (202) 336-6020

\section{Regionals}

Eastern Psychological Association

April 15-18, 2004, Washington, DC

For information

Roberta E. Dihoff

Department of Psychology

Rowan University

Glassboro, NJ 08028

Telephone: (856) 256-4500 ext. 3783

E-mail: epaex@aol.com

Midwestern Psychological Association May 8-10, 2003, Palmer House Hilton,

Chicago, IL

For information

Ralph Erber, Chair

Department of Psychology

DePaul University

2219 North Kenmore

Chicago, IL 60614

Telephone: (773) 325-4243

New England Psychological Association

November 7-8, 2003, Salem, MA

For information

Nancy Jackson

Johnson and Wales University

8 Abbott Park Place

Providence, RI 02903

Rocky Mountain Psychological Association

April 15-17, 2004, Reno, NV

For information

Nancy J. Karlin

Department of Psychology

McKee 14

University of Northern Colorado

Greeley, CO 80639

E-mail: njkarli@unco.edu
Southeastern Psychological Association

March 10-13, 2004, Atlanta Hilton,

Atlanta, GA

For information

Rosemary Hays-Thomas

Department of Psychology

University of West Florida

Pensacola, FL 32514

Telephone: (850) 474-2070

Southwestern Psychological Association April 8-11, 2004, San Antonio, TX

For information

Raymond Russin

Department of Psychology

Bethany College

421 North First Street

Lindsborg, KS 67456

Southern Society for Philosophy and

Psychology

April 8-10, 2004

For information

David A. Washburn

Department of Psychology

Georgia State University

Atlanta, GA 30303

Telephone: (404) 244-5845

E-mail:dwashburn@gsu.edu

Western Psychological Association

April 8-11, 2004, San Antonio, TX

For information

http://www.westernpsych.org

or

Donald E. Pannen

WPA Executive Officer

5929 Westgate Boulevard, Suite C

Tacoma, WA 98406

Telephone: (253) 752-9838

Fax: (253) 752-9829

E-mail: pannen.wpa@worldnet.att.net

\section{Other}

American Association for the

Advancement of Science

February 12-17, 2004, Seattle, WA

American Educational Research Association April 12-16, 2004, San Diego, CA

American Psychological Society May 27-30, 2004, Chicago, IL

American Psychology-Law Society

(Division 41)/Psychology and Law
International Interdisciplinary Conference July 7-12, 2003, Edinburgh, Scotland March 2004, La Jolla, CA

Animal Behavior Society July 15-23, 2003, Boise, ID

Association for the Advancement of Behavior Therapy

November 20-23, 2003, Boston, MA

Association for Behavior Analysis

May 23-27, 2003, San Francisco, CA

Human Factors and Ergonomics Society October 13-17, 2003, Denver, CO

National Council on Measurement in Education

April 21-25, 2003, Chicago, IL

Psychonomic Society

November 6-9, 2003, Vancouver, British Columbia, Canada

Society for Industrial and Organizational Psychology (Division 14)

April 2-4, 2004, Chicago, IL

Society for Neuroscience

November 8-12, 2003, New Orleans, LA

Society for Personality and Social

Psychology (Division 8)

January 29-31, 2004, Austin, TX

Society for the Psychological Study of Social Issues (Division 9)

June 25-27, 2004, Washington, DC

Society of Behavioral Medicine March 24-27, 2004, Baltimore, MD

\section{International Meetings}

A more extensive listing of international meetings can be found in the International Journal of Psychology, available at many libraries, from Psychology Press, or at http:// aix1.uottawa.ca/ iupsys/mtg.html. In addition, your reference librarian should be able to direct you to other specialized meeting calendars.

Eleventh Annual International Conference on Conflict Resolution: Sharing Tools for Personal/Global Harmony

May 8-18, 2003, St. Petersburg, Russia 
For information

Common Bond Institute (USA)

Steve Olweean, Conference Coordinator

12170 S. Pine Ayr Drive

Climax, MI 49034

Telephone/Fax: (269) 665-9393

E-mail: solweean@aol.com

Web site: http://ahpweb.org/cbi/home.html

Second International Conference on

Violence in School

May 11-14, 2003, Québec City, Québec, Canada

For information

Égide Royer

Faculté des sciences de l'éducation

Université Laval

c/o Québec 2003 Secretariat

Agora Communication

2600 boulevard Laurier

bur. 2680

Sainte-Foy, QC G1V 4M6

Canada

Telephone: (418) 658-6755

Fax: (418) 658-8850

E-mail: quebec2003@agoracom.qc.ca

Web site: http://www.ulaval.ca/crires/

Eleventh European Congress on Work and Organizational Psychology

May 14-17, 2003, Lisbon, Portugal

For information

Antonio Caetano, Chairman of the Organizing

Committee and Associate Professor

Department of Social and Organizational Psychology

ISCTE-Instituto Superior de Ciencias do

Trabalho e da Empresa

Av. das Forcas Armadas

Edif ISCTE

1649-026 Lisboa, Portugal

E-mail: eawopcongress@iscte.pt

Web site: http://www.eawop-congress.iscte.pt

Eighth European Conference on Traumatic Stress

May 22-25, 2003, Berlin, Germany

For information

VIII ECOTS Berlin 2003

CPO Hanser Service GmbH

P.O. Box 330316

D-14173 Berlin, Germany

Telephone: 49-30-300 6690

Fax: 49-30-305 7391

E-mail: berlin@cpo-hanser.de

Web site: http://www.trauma-conference-

berlin.de

Third International Conference on Psychology and Ergonomics: The Unity of Theory and Practice

June 5-8, 2003, Moscow and Tver, Russia

For information

Conference Secretariat

Korp. 4, Khrustalnaya U1.2

Tver, Russia, 17001

E-mail: ergocentre@tvcom.ru

Sixth International Symposium on

Pediatric Pain

June 15-19, 2003, Sydney, Australia

For information

DC Conferences
P.O. Box 571

Crows Nest, NSW 1585

Australia

Telephone: 61294396744

Fax: 61294392504

E-mail: ispp2003@dcconferences.com.au

or

mail@dcconferences.com.au

Second World Congress on Family

Violence

June 21-26, 2003, Prague, Czech Republic

For information

International Network on Family Violence

1025 Connecticut Avenue, NW, Suite 1012

Washington, DC 20026

Telephone: (202) 429-6695

Fax: (831) 655-3930

E-mail:wcfv@aol.com

Web site: http://www.familyviolence.org

Tenth Biennial Conference of the

International Society for Theoretical

Psychology

June 22-27, 2003, Istanbul, Turkey

For information

Aydan Gulerce, Chair

ISTP2003 Organizing Committee

Bogazici University

Bebek, Istanbul, 80815

Turkey

Fax: (90) 2122575036

E-mail: istp2003@boun.edu.tr

Eighth European Congress of Psychology-

Psychology in Dialogue With Related

Disciplines

July 6-11, 2003, Vienna, Austria

For information

Monika Glantschnig

EFPPA 2003 Committee

Austrian Professional Association of

Psychologists

Möllwaldplatz 4/4/39

A-1040 Vienna

Austria

Telephone: $43 \quad 14072671 \quad 17$

Fax: 431407267130

E-mail: info@psycongress.at

Web site: http://www.psycongress.at

Psychology and Law International

Interdisciplinary Conference 2003

July 7-12, 2003, Edinburgh, Scotland

For information

Jill Elliott, Conference Administrator

BS\&L Network

Faculty of Law

University of Southampton

Southampton

Hampshire SO17 1BJ

United Kingdom

Telephone: 44 (0) 2380592376

Fax: 44 (0) $23 \quad 80593884$

E-mail: jill.elliott@soton.ac.uk

Web site: http://www.law.soton.ac.uk/bsln/ psych\&law2003

Sixth European Regional Congress of the International Association for Cross-Cultural Psychology

July $12-16,2003$, Budapest, Hungary
For information

Marta Fulop

MTA Pszichologiai Kutatointezet

Victor Hugo utca 18-22

Budapest, Hungary-1132

E-mail: fmarta@mtapi.hu

Web site: http://www.psychology.hu/iaccp

Twenty-Ninth Interamerican Congress of Psychology

July 13-18, 2003, Lima, Peru

For information

E-mail: sip2003peru@terra.com

Web site: http://www.sip2003.org

Fourth Annual Couples Therapy Training Workshop for Therapists

July 13-18, 2003, near Prague, Czech Republic

For information

Rita Resnick

1460 7th Street, \#300

Santa Monica, CA 90401

Telephone: (310) 395-6844

Fax: (310) 395-6844

E-mail: SweetRita@aol.com

Web site: http://www.CouplesTherapyTraining .com

Thirty-Second Annual Gestalt Therapy European Summer Residential Training Program

July 20-August 1, 2003, near Prague, Czech Republic

For information

Rita Resnick

1460 7th Street, \#300

Santa Monica, CA 90401

Telephone: (310) 395-6844

Fax: (310) 395-6844

E-mail: SweetRita@aol.com

Web site: http://www.GATLA.org

Eighth Minnesota International Counseling Institute

July 27-August 1, 2003, Minneapolis, MN

For information

Thomas Skovholt

Minnesota International Counseling Institute

University of Minnesota

129 Burton Hall

178 Pillsbury Drive, SE

Minneapolis, MN 55455

E-mail: cspp-adm@tc.umn.edu

Web site: http://www.education.umn.edu/ EdPsych/CSPP/mici

2003 International Rudolf Dreikurs

Summer School

July 27-August 8, 2003, Guelph, Ontario, Canada

For information

Mike Balla, Program Administrator

E-mail: mj.balla@sympatico.ca

Web site: http://www.icassi.org

International Council of Psychologists 61st Annual Conference

August 11-14, 2003, Toronto, Ontario, Canada

For information

Edith H. Grotberg

Georgetown University School of Nursing

and Health Studies 
Box 571107

Washington, DC 20057

Telephone: (202) 687-3332

E-mail: eg96@georgetown.edu

Web site: http://icpsych.tripod.com

Eleventh International Congress of the International Psychogeriatric Association August 17-22, 2003, Chicago, IL

For information

Secretariat, International Psychogeriatric

Association

5215 Old Orchard Road, Suite 340

Skokie, IL 60077

Telephone: (847) 663-0574

Fax: (847) 663-0591

E-mail: chicago2003@ipa-online.org

Web site: http://www.ipa-online.org

Fourteenth International Symposium for the Psychological Treatment of Schizophrenia and Other Psychoses

September 22-25, 2003, Melbourne,

Victoria, Australia

For information

ISPS 2003 Secretariat

Telephone: 61395218881

Fax: 61395219521

E-mail: isps@conferencestrategy.com.au

Web site: http://www.conferencestrategy.com.au isps/index.html

International Conference on War and

Peace: Rethinking Gender, War, and

Peace-Feminist Perspectives

October 10-12, 2003, Washington, DC

For information

Martha Mednick and Anne Anderson

Psychologists for Social Responsibility

2604 Connecticut Avenue, NW

Washington, DC 20008

Telephone: (202) 745-7084

Fax: (202) 754-0051

Web site: http://www.psysr.org
Twentieth International Conference of the International Society for Quality in Health Care

November 2-5, 2003, Dallas, Texas

For information

ISQua Congress Secretariat, Level 9

Aikenhead Center

27 Victoria Parade

Fitzroy, Victoria 3065

Australia

Telephone: 61394176971

Fax: 61394176851

E-mail: isqua@isqua.org.au

Web site: http://www.isqua.org.au

Fifth Conference of the European

Academy of Occupational Health

Psychology: Flexibility, Quality of

Working Life, and Health

November 20-21, 2003, Berlin, Germany

For information

European Academy of Occupational Health Psychology

E-mail: conferences@ea-ohp.org

Web site: http://www.ea-ohp.org

Middle East/North Africa Regional

Conference on Psychology (MENA RCP)

December 13-18, 2003, Dubai, United Arab Emirates

For information

Raymond H. Hamden, President

MENA RCP

P.O. Box 11806

Dubai

United Arab Emirates

Telephone: (971.4) 331.4777

E-mail: info@menarcp.org

Third International Congress on

Licensure Certification and

Credentialing of Psychologists

Hosted by The Association of State and

Provincial Psychology Boards
April 21-24, 2004, Montreal, Quebec, Canada

For information

ASPPB - 3rd International Congress

Mary E. Willmuth, Secretary General

P.O. Box 241245

Montgomery, AL 36117-1245

E-mail: 3icinfo@asppb.org

Web site: http://www.asppb.org/congress

Seventeenth Congress of the International Association for Cross-Cultural Psychology (IACCP)

August 2-6, 2004, Xian, China

For information

IACCP 2004

c/o The Chinese Psychological Society

10A Datun Road

Beijing 100101

China

E-mail: iaccp2004@psych.ac.cn

Web site: http://www.iaccp.org

Twenty-Eighth International Congress of Psychology

August 8-13, 2004, Bejing, China

For information

ICP 2004

c/o The Chinese Psychological Society

10A Datun Road

Beijing 100101

China

E-mail: icp2004@psych.ac.cn

Web site: http://www.icp2004.psych.ac.cn

Sixth Delphi International Psychoanalytic Symposium

October 27-31, 2004, Patras, Greece

For information

Eleni Vouga

Department of Psychiatry

University of Patras

26500 Patras

Greece

Fax: 0610-994.535

E-mail: delphi@med.upatras.gr 